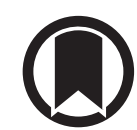

CrossMark

\section{Increased ventilation heterogeneity in asthma can be attributed to proximal bronchioles}

To the Editor:

In recent years, tests of ventilation heterogeneity such as the multiple breath washout (MBW) have gained popularity as potentially sensitive measures of disease in the small airways [1]. The premise of a non-invasive test, such as the MBW, is that morphological alterations at differing lung depths can be sampled at the mouth via their effect on ventilation heterogeneity. MBW phase III slope derived indices of ventilation heterogeneity include the conductive slope index (Scond), a purely convection-dependent contribution generated in the conducting zone of the lungs, and the acinar slope index (Sacin), brought about by diffusion-convection mechanisms operating near acinar entrances in the respiratory zone [2, 3].

Sacin and Scond hold promise as clinical monitoring tools in asthma $[4,5]$. In severe asthma, Sacin has been shown to become abnormally elevated [6], and partly linked to peripheral eosinophilic inflammation [7]. However, across the clinical spectrum of presented asthma cases, Scond stands out as being consistently elevated, and strongly contributing to airway hyperresponsiveness, independent of airway inflammation [8]. This prompts a clinical question: what is the discriminating structural feature that causes elevation of Scond in asthmatic lungs? State-of-art ventilation imaging modalities indicate increased convection-dependent ventilation heterogeneity in asthma $[9,10]$, but these do not preclude the possibility that Scond is partially or predominantly generated beyond imaging resolution.

For the conductive portion of the phase III slope (represented by Scond) to be positive at a given branch point in the airway tree, two conditions need to be fulfilled [2]: 1) heterogeneous specific ventilation between the two subtended lung units supplied by convective flow, resulting in heterogeneous washout gas concentration; and 2) any expiratory flow pattern where the least ventilated lung unit has a contribution to total expiratory flow at end of expiration which is greater than its contribution at the beginning of expiration (with balance expiratory flow from the other unit). Given these conditions, it is impossible to infer a priori whether elevation in Scond is primarily generated proximally or distally within the conducting lung zone. In healthy adults, Scond can be partly accounted for by temporal expansion differences between lobes, as observed by dynamic computed tomography (CT) [11]. However, in asthmatic patients, computational gas transport modelling on patient-based airway structures has accurately reproduced the experimentally measured Scond increases, based solely on their airway structure [12]. This offers the possibility to scrutinise the specific bronchial generations where airway alterations are responsible for the observed Scond elevation in asthma.

We retrieved inspiratory CT-derived, patient-based lung structures of 24 asthmatic subjects (15 male; mean \pm SD $50 \pm 13$ years; forced expiratory volume $1 \mathrm{~s}\left(\mathrm{FEV}_{1}\right)$ /forced vital capacity $0.69 \pm 0.08 ; \mathrm{FEV}_{1} 81 \pm 19 \%$ predicted; ACQ-6 2.02 \pm 0.87 ) from a previous study [13] without modifying the geometry. In these structures, airways below CT resolution (typically beyond airway generation 7 , which was on average $92 \%$ complete), had been grown algorithmically within the identified lobar boundaries, with daughter airways having radii scaled from their parent. In a prior study, we used a fluid flow model dictated by radii and lengths (resistance) of all airways and a fixed compliance of all terminal units (acini), and incorporating a gravity-dependent pleural pressure gradient, to simulate $\mathrm{SF}_{6}-\mathrm{MBW}$ tests in an upright position. This resulted in a strong concordance between experimental and simulated Scond for these 24 asthma patients [12].

@ERSpublications

By combining patient-specific CT-based airways with a clinically validated lung model, the proximal bronchioles were identified as the airways where structural abnormality can explain the increased convective ventilation heterogeneity in asthma patients $\mathrm{http}: / / \mathrm{bit} . l \mathrm{ly} / 2 \mathrm{COT} 0 \mathrm{Qh}$

Cite this article as: Foy B, Kay D, Siddiqui S, et al. Increased ventilation heterogeneity in asthma can be attributed to proximal bronchioles. Eur Respir J 2020; 55: 1901345 [https://doi.org/10.1183/ 13993003.01345-2019]. 
By design, the model geometry incorporated simplified compliant acinar units without internal structure, and hence there is no diffusion-convection heterogeneity contribution to the phase III slope. Therefore, studying the mechanisms of convection-dependent ventilation heterogeneity with this model, and quantified by Scond in MBW experiments, reduces to examination of the phase III slope of the first exhalation [2].

We simulated $\mathrm{SF}_{6}$ concentrations and airflow profiles at every branch point in the lung, over the entire inand exhalation of the first breath. This allowed us to calculate a single-breath phase III slope for each branch in each lung structure, instead of just at the mouth. By combining phase III slopes from all parallel branch points, the average phase III slope contribution at any airway generation, relative to that obtained at the patient's mouth, could be calculated.

Averaged over 24 patients, the coefficient of variation of washout gas concentration $(\mathrm{CoV}(\mathrm{conc}))$ between any parallel pair of lung units (figure 1a) and corresponding phase III slope (figure 1b) illustrates several striking features. Firstly, over $80 \%$ of the phase III slope appears to be generated in generations $5-9$, with most of this occurring in generations 5-7. Secondly, despite considerable concentration heterogeneity in generations $1-4$, there is little effect on phase III slope. Furthermore, generations 10 onwards only marginally contribute to the phase III slope. It can thus be concluded that, in asthmatic subjects,
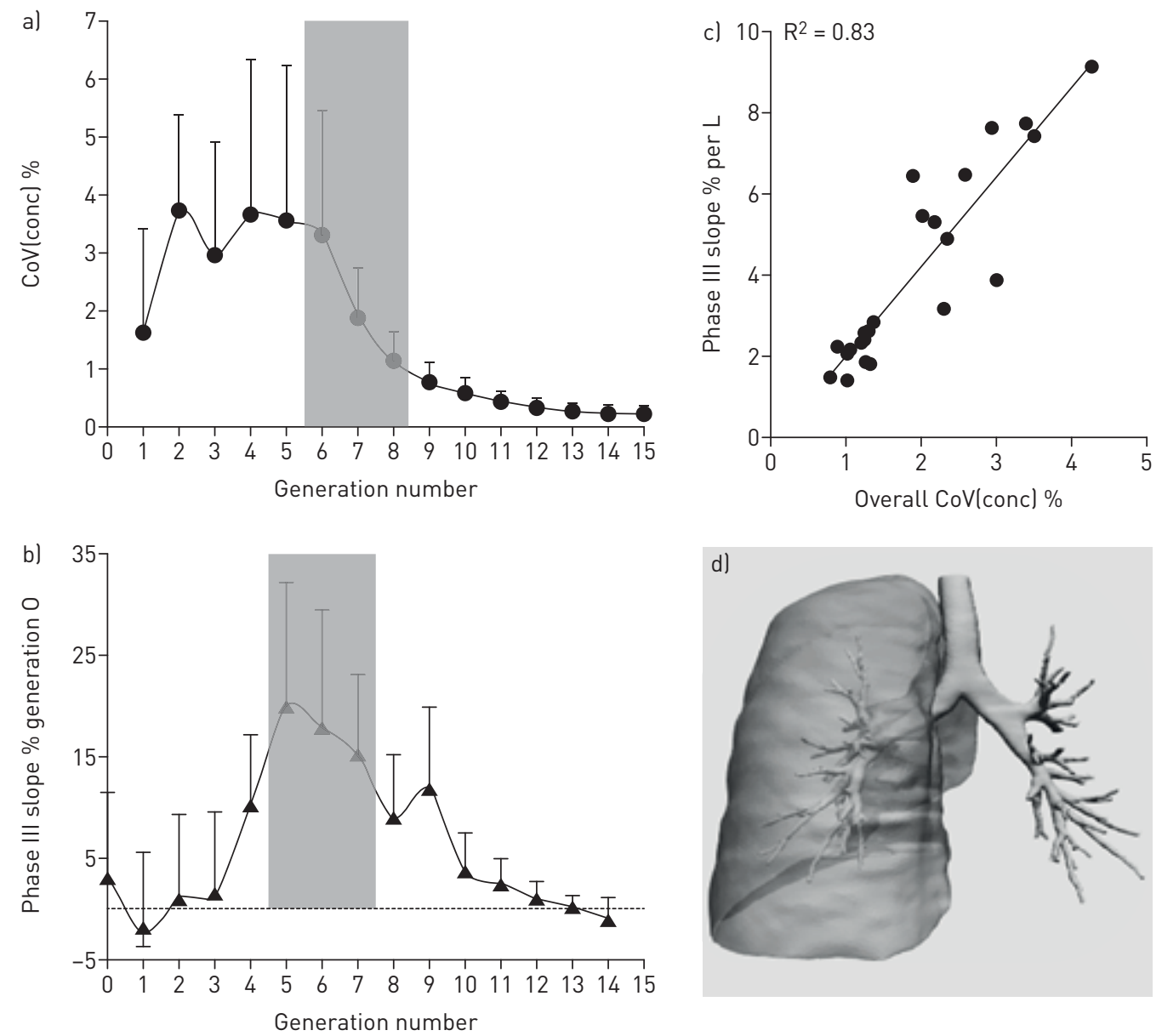

FIGURE 1 Concentration heterogeneity and phase-III slope. a) Generation-specific concentration coefficient of variation (CoV(conc)) as a function of airway generation number; data obtained from all 24 lung structures is presented as mean+SD; grey shaded area indicates the generation range responsible for maximal phase III slope contribution in b. Note that the phase III slope in any generation is driven by CoV(conc) in the next (more distal) generation, resulting in a shift between both plots by one generation. b) Generation-specific phase III slope (relative to phase III slope at the mouth) as a function of airway generation number; data obtained from all 24 lung structures is presented as mean+SD; grey shaded area indicates the generation range responsible for maximal phase III slope contribution. c) Phase III slope at the mouth is tightly correlated with overall concentration heterogeneity (Pearson $\mathrm{R}^{2}=0.83$ ). d) An example of one reconstructed patient-derived lung structure is shown, with computed tomography (CT)-extracted airways and lung boundaries shown for the right lung (left side of image), and CT-extracted airways shown for the left lung lalgorithmically generated airways have not been included for clarity). 
the elevation in Scond can be primarily attributed to the proximal bronchiole typically within the segmentation range of CT imaging. Finally, irrespective of the relative contribution from different lung depths to phase III slope at the mouth, the latter is mainly determined by overall level of concentration heterogeneity, with $83 \%$ of variability explained, as can be inferred from figure 1c. Finally, we also imposed, in two representative structures, an additional random cross sectional heterogeneity ( $\mathrm{SD}=0.2)$ in generations 6- or 10- onwards, which led to minor increases in $\mathrm{CoV}$ (conc) and no consistent phase III slope contributions in these airway generations (data not shown). Note that any such contribution would have come as an add-on to the phase III slopes we obtain here based on contributions from airway generations 4-7 with actual airway dimensions segmented from CT and which suffice to explain experimental observations [12].

In the clinic, the two main physiological tests of small airways function for which European Respiratory Society guidelines exist, are airway mechanics measurements by forced oscillation and tests of ventilation heterogeneity by multiple breath washout. Both techniques have a similar drawback in that different combinations of airway alterations can potentially lead to the same numeric value of indices measured at the mouth. Via simulations with a realistic lung model [13], it is possible to narrow down the number of combinations inside the lungs that can account for the indices at the mouth. This is what we have achieved with the present study, where we identified the lung depth where airway structure is likely to cause the Scond increases seen in asthma. While from first principles [2], Scond can be generated in all conductive airway generations including the small airways, our data clearly show that in asthma, it is the proximal bronchioles that account for an elevated Scond. This ties in with an independent modelling study [14] where it is shown that proximal airways may be responsible for an avalanche-type flow redistribution compatible with patterns of ventilation defects observed in asthma $[9,10]$. Also important for the clinical utility of our findings is that our $\mathrm{SF}_{6}-\mathrm{MBW}$ simulations also apply to $\mathrm{N}_{2}$-MBW [15], because for Scond only diffusion front location matters [2]. The respective diffusion fronts being merely a generation apart, both their location is also far more peripheral than where Scond is now shown to be generated.

We conclude that in asthma, parallel heterogeneity of airway calibre and length within the proximal bronchioles is the discriminating structural feature that can be picked up by non-invasive MBW measurement of conductive ventilation heterogeneity, in the presence of flow sequencing. Bronchial generations 5-7 show the greatest relative contribution to Scond, with the extent of Scond abnormality being dictated by the degree of concentration heterogeneity. While it is possible that the degree of concentration heterogeneity (typically 5\%) could also be picked up with quantitative ventilation imaging, our work illustrates that the MBW index Scond is a valid detection alternative.

Brody Foy $\oplus^{1,2}$, David Kay ${ }^{3}$, Salman Siddiqui ${ }^{4}$, Chris Brightling ${ }^{4}$, Manuel Paiva ${ }^{5}$ and Sylvia Verbanck ${ }^{6}$

${ }^{1}$ Center for Systems Biology and Dept of Pathology, Massachusetts General Hospital, Boston, MA, USA. ${ }^{2}$ Dept of Systems Biology, Harvard Medical School, Boston, MA, USA. ${ }^{3}$ Dept of Computer Science, University of Oxford, Oxford, UK. ${ }^{4}$ Dept of Respiratory Sciences, University of Leicester, Leicester, UK. ${ }^{5}$ Respiratory Division, University Hospital Erasme, Brussels, Belgium. ${ }^{6}$ Respiratory Division, University Hospital UZBrussel, Brussels, Belgium.

Correspondence: Sylvia Verbanck, Respiratory Division, University Hospital UZ Brussel, Laarbeeklaan 101, 1090 Brussels, Belgium. E-mail: sylvia.verbanck@uzbrussel.be

Received: 08 July 2019 | Accepted after revision: 12 Nov 2019

Author contributions: B. Foy helped conceive the study, developed the computational model, performed simulations, analysed the data and co-wrote the manuscript. D. Kay supervised the creation of the computational model, provided a scientific critique of the data and edited the manuscript. S. Siddiqui and C. Brightling provided clinical interpretation of the data, oversaw CT segmentation and airway centreline generation and edited the manuscript. M. Paiva helped analyse the data, provided a scientific critique of the data, and edited the manuscript. S. Verbanck conceived the study, analysed the data, and co-wrote the manuscript.

Conflict of interest: B. Foy has nothing to disclose. D. Kay has nothing to disclose. S. Siddiqui has nothing to disclose. C. Brightling reports grants and personal fees from GSK, AZ/MedImmune, Novartis, Chiesi, Pfizer, Vectura, Theravance, BI and Roche/Genetech, outside the submitted work. M. Paiva has nothing to disclose. S. Verbanck has nothing to disclose.

Support statement: This project was supported by the Fund for Scientific Research-Flanders (FWO-Vlaanderen, Belgium). Generation of the virtual lung structures was supported by an EU Framework 7 project grant (number 270194). Partially funded by Novartis UK. Funding information for this article has been deposited with the Crossref Funder Registry.

\section{References}

1 Robinson PD, Latzin P, Verbanck S, et al. Consensus statement for inert gas washout measurement using multiple- and single- breath tests. Eur Respir J 2013; 41: 507-522. 
Verbanck S, Paiva M. Gas mixing in the airways and airspaces. Compreh Physiol 2011; 1: 809-834.

Verbanck S, Paiva M. A simulation study of diffusion-convection interaction and its effect on multiple breath washout indices. Respir Physiol Neurobiol 2018; 258: 5-11.

4 Farah CS, King GG, Brown NJ, et al. The role of the small airways in the clinical expression of asthma in adults. J Allergy Clin Immunol 2012; 129: 381-387.

5 Postma DS, Brightling C, Baldi S, et al. Exploring the relevance and extent of small airways dysfunction in asthma (ATLANTIS): baseline data from a prospective cohort study. Lancet Respir Med 2019; 7: 402-416.

6 Gonem S, Hardy S, Buhl N, et al. Characterization of acinar airspace involvement in asthmatic patients by using inert gas washout and hyperpolarized ${ }^{3}$ helium magnetic resonance. J Allergy Clin Immunol 2016; 137: 417-425.

7 Verbanck S, Schuermans D, Vincken W. Inflammation and airway function in the lung periphery of patients with stable asthma. J Allergy Clin Immunol 2010; 125: 611-616.

8 Downie SR, Salome CM, Verbanck S, et al. Ventilation heterogeneity is a major determinant of airway hyperresponsiveness in asthma, independent of airway inflammation. Thorax 2007; 62: 684-689.

9 Svenningsen S, Nair P, Guo F, et al. Is ventilation heterogeneity related to asthma control? Eur Respir J 2016; 48: 370-379.

10 Farrow CE, Salome CM, Harris BE, et al. Peripheral ventilation heterogeneity determines the extent of bronchoconstriction in asthma. J Appl Physiol 2017; 123: 1188-1194.

11 Verbanck S, Paiva M. Could lobar flow sequencing account for convection-dependent ventilation heterogeneity in normal humans? J Appl Physiol 2016; 121: 589-591.

12 Foy BH, Gonem S, Brightling C, et al. Modelling the effect of gravity on inert-gas washout outputs. Phys Rep 2018; 6: e13709.

13 Bordas R, Lefevre C, Veeckmans B, et al. Development and analysis of patient-based complete conducting airway models. PLoS One 2015; 10: e0144105.

14 Donovan GM, Elliot JG, Green FHY, et al. Unraveling a clinical paradox: why does bronchial thermoplasty work in asthma? Am J Respir Cell Mol Biol 2018; 59: 355-362.

15 Foy B, Kay D. A computationally tractable scheme for simulation of the human pulmonary system. J Comput Phys 2019; 388: 371-393. 